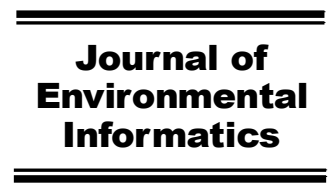

www.iseis.org/jei

\title{
DCT-Based Least-Squares Predictive Model for Hourly AQI Fluctuation Forecasting
}

\author{
Z. C. Yang ${ }^{1 *}$ \\ ${ }^{1}$ School of Information and Electrical Engineering, Hunan University of Science and Technology, Xiangtan 411201, China
}

Received 6 June 2017; revised 26 July 2018; accepted 30 July 2018; published online 6 April 2019

\begin{abstract}
Recently the issue of air quality has become a global public health concern. As air pollution has been reported as the largest single environmental health risk in the world, analysis and prediction of air quality is increasingly important. Normally, either statistical models or CTM ("deterministic chemistry-transport") models are used for forecasting air PM ("particulate matter") levels. Actually, hourly air quality fluctuation is also one time-series. Compared with commonly used deterministic photochemical air quality models, data-driven or time-series-based modeling is simpler and can also perform well even be more accurate. In this study, a called DCT(“discrete cosine transform")-based least-squares predictive model is proposed for forecasting hourly AQI ("air quality index") from time-series analysis or data-driven modeling perspective. The proposed DCT-based predictive method is implemented in combination with the least-squares method to compute the called least-squares-optimum DCT coefficients for forecast modeling on the basis of finite hourly AQI observations. The proposed method yields one good result of average $93.24 \%$ predictive accuracy in forecast experiments at five monitoring stations in Xiangtan of China. Experimental results and analysis of performance comparisons of the proposed DCT-based least-squares predictive model with the classical BP-ANN model, the Fourier-series-based least-squares predictive model and the ARIMA model indicate that for the same tasks of forecasting hourly AQI fluctuations, the proposed DCT-based predictive model outperforms the former two competitive models and performs slightly better than or comparable to the ARIMA model. It is indicated that the hourly AQI fluctuations can be well forecasted by the proposed DCT-based least-squares predictive model with using about 12-term least-squares-optimum DCT coefficients.
\end{abstract}

Keywords: DCT-based model, forecasting, hourly AQI, least-squares fitting

\section{Introduction}

Air quality is intimately associated with human health. Nowadays air pollution has been recognized as a major source of health risk (Briggs, 2003) and one of the significant factors to the environmental burden of disease throughout the world. Human (and animal) health will be affected by exposure to high concentrations of air pollutants. Recently, it is reported by the World Health Organization (WHO, 2014) that as one result of air pollution exposure, around 7 million people died in 2012, which became one in eight of total global deaths. Thus air pollution has now been recognized as the largest single environmental health risk in the world (WHO, 2014). Accordingly, the issue of air quality has become one public concern (Yang, 2014). The AQI ("air quality index"), also known as the API ("air pollution index"), the PSI ("pollutant standard index") (Cheng et al., 2007) and the AQHI ("air quality health index)" (Chen et al., 2013; Wong et al., 2013), is a quantity commonly used for describing air quality. It is an index for

\footnotetext{
${ }^{*}$ Corresponding author. Tel.: +086130472211172
}

E-mail address: yzc233@163.com (Z. C. Yang).

ISSN: $1726-2135$ print/1684-8799 online

(C) 2018 ISEIS All rights reserved. doi:10.3808/jei.201800402 reporting quality of ambient air at one location and severity level of air pollution to the public. Generally, the higher AQI, the higher probability of the public affected by adverse health effects will be. Addressing limitations of the conventional AQI such as high levels of subjectivity, Sowlat et al. (2011) presented a new fuzzy-logic-based AQI called the FAQI. In view of adverse effects caused by the coexistence of all the pollutants, Plaia et al. (2013) proposed one called multi-pollutant-multisite API. For evaluating the air quality impact of environmental policies, Fassò (2013) presented an EM-algorithmbased spatio-temporal model. Benis et al. (2013) designed one multi-objective and multi-pollutant sensitive AQMN ("air quality monitoring net work") using an integrated optimization approach. For the regional air pollution management and control, Liu et al. (2015) presented a called ISRAQM ("inexact stochastic robust air quality management") model. We see that the AQI, which is an index for describing air quality and easy for the public to understand, has been one of daily concerns of the general public. Accordingly, analysis and prediction of air quality becomes increasingly important.

Human actives and meteorological factors may largely affect the particle pollution in the air. Normally, either statistical models or CTM ("deterministic chemistry-transport") models are used for forecasting the air PM ("particulate matter") levels (Konovalov et al., 2009). Actually, hourly air qual- 
ity fluctuation is also one time-series. The RT-AQF ("real-time air quality forecasting") (Zhang et al., 2012) has been a new inter-disciplinary research with unprecedented scientific, technical, and computational challenges being proposed. Since the 1970s, a variety of RT-AQF techniques and tools have been employed to predict air pollution in urban areas. Among the various techniques, empirical approaches and statistical models based on historical air quality and meteorological data are the representative ones. From the viewpoint of time-series analysis or data-driven modeling, mathematical methods (Chatterjee et al., 2000; Chatfield, 2004; StatSoft, 2013) used for time-series analysis mainly include the classical time-series analysis technique in statistics and the computational intelligence. The classical time-series analysis is one standard statistical technique. The AR ("autoregressive") model, the MA ("moving average") model, the ARMA ("autoregressivemoving-average") model, and the ARIMA ("autoregressive integrated moving average") model are classical statistical models in this direction. Stationarity is an important assumption in the classical time-series analysis technique. That is, the underlying stochastic process is assumed to be stationary, and then the process may be adequately depicted by the lower moments of its probability distribution. For daily AQI predicttion, Kumar and Goyal (2011) tested three statistical models (the ARIMA, PCR ("principal component regression") and one combination model of both) and indicated that the last combination model performed better than the former two ones. ANN ("artificial neural network") is one of the most popular and promising subjects in artificial and computational intelligence areas. It has been pervasively used in various applications with well forecast performances being reported. He and Deng (2012) presented one hybrid model of ARIMA and ANN to predict the PM concentration in Changsha of China, and showed that compared with the single ARIMA model, the hybrid model improved the $\mathrm{PM}_{10}$ prediction accuracy. Rahman et al. (2013) used the ARIMA, ANN and FTS ("fuzzy time-series") for predicting API values in Malaysia, and showed that the ANN performed better than the FTS and the ARIMA. Kolehmainen et al. (2001) evaluated two principally different ANNs in three ways related to periodic components, and showed that compared with the ways of only using the periodic components or removing periodic components, best forecast results were achieved by the way of directly using a multi-layer ANN and the original data, and then concluded that combinations of the periodic regression method and neural algorithms did not exhibit any advantage over the direct application of neural algorithms. Niska et al. (2004) presented at a study on hourly $\mathrm{NO}_{2}$ concentration prediction at one busy urban traffic monitoring station by using a multilayer ANN combined with a parallel GA ("genetic algorithm"), and then argued the limitation of the ANN technique that evaluation of an ANN model was a computationally expensive step. On the basis of 3 statistical criteria, Kukkonen et al. (2003) evaluated five ANN models, a linear statistical model and a deterministic modeling system for forecasting urban $\mathrm{NO}_{2}$ and $\mathrm{PM}_{10}$ concentrations, and then argued that the currently available ANN models were neither applicable for fore- casting spatial concentration distributions in urban areas, nor for evaluating air pollution abatement scenarios for future years. Grell and Baklanov (2011) argued that integrated modeling systems, which allow two-way interactions of physical and chemical processes, would provide new opportunities for the migration of weather and air quality prediction. Nevertheless, Benis et al. (2013) argued that with meaningful advances in computational technologies, a new focus would be shifted to sophisticated 3-D RT-AQF models (Zhang et al., 2012), which are expected to enhance people's understanding of the underlying complex interplay of meteorology, emission, and chemistry from global to urban scales in the real atmosphere.

In this study, a DCT-based least-squares predictive model is proposed for hourly AQI forecasting from the viewpoint of time-series analysis or data-driven modeling. Compared with commonly used deterministic photochemical air quality models, data-driven or time-series-based models are simpler and can also perform well even be more accurate (Sun et al., 2013). From the perspective of time-series analysis or data-driven modeling, fluctuations of daily 24-hour air quality, which may be influenced by various factors, can also be described as one timeseries. Then there are two basic goals included in its timeseries analysis (StatSoft, 2013): (a) try to reveal nature of the phenomenon depicted by the observation sequence, and (b) try to predict future fluctuations on the basis of the observations available. Fourier-based technology is a powerful tool for timeseries analysis. DCT refers to "discrete cosine transform". It is also a Fourier-related transform. This transform technique has been widely used in signal and image processing (Ge et al., 2008). It owns remarkable properties on optimal decorrelation and energy compaction, asymptotically approaching the KLT ("Karhunen-Loève transform") (Zheng et al., 2011). However, Fourier-based analysis in its conventional form cannot be straightly applied to prediction (Yang, 2013, 2014). In this study, a DCT-based predictive model extended in the least-squares sense is introduced for hourly AQI fluctuation prediction. The DCT-based least-squares forecast modeling, which was applied to the electric load forecasting (Yang, 2016) and the hourly WLF ("water-level-fluctuation") forecasting (Yang, 2017), is implemented in combination with the least-squares method to compute the called least-squares-optimum DCT coefficients on the basis of finite hourly AQI observations. Finally, experiments and result analysis are also presented. Experimental result and performance analysis of the proposed DCT-based leastsquares predictive model with other competitive models indicate potentiality of the proposed model. It is indicated that concise and well-formed may be one major advantage of the proposed DCT-based least-squares predictive model for hourly AQI forecasting.

\section{The Proposed Methodology}

\subsection{Problem Description}

Fluctuation of daily 24-hour air quality, which may be influenced by varied factors, can also be described as one timeseries. DCT is a Fourier-related transform (Zheng et al., 2011). The most common 1-D ("one-dimensional") DCT definition 
for a sequence (discrete time-series) $x(n)$ of length $N(n=1,2$, $\ldots, N)$ is defined as:

$$
f(k)=w(k) \sum_{n=1}^{N} x(n) \cos \left[\frac{\pi(2 n-1)(k-1)}{2 N}\right]
$$

The transform coefficients of $f(k)(n=1,2, \ldots, N)$ (Equation (1)) are called the DCT coefficients. Similarly, the inverse DCT (IDCT) is defined as:

$$
x(n)=\sum_{k=1}^{N} f(k) w(k) \cos \left[\frac{\pi(2 n-1)(k-1)}{2 N}\right]
$$

In the literature, the value of $f(k)(k=1)$ may be referred to as the DC Coefficient and the other ones of $f(k)(k=2, \ldots$, $N)$ are called the AC Coefficients (Sayood and Borkenhagen, 1991).

From the above definitions (Equations 1 and 2), it is indicated that both the sequence (discrete time-series) $x(n)(n=1$, $2, \ldots, N)$ and the DCT transform coefficients: $f(k)(k=1,2$, $\ldots, N)$ are the same size of $N$-length. If only a limited number $N_{0}(<N)$ of hourly AQI observations over a certain period time: $x(n)\left(n=1,2, \ldots, N_{0}\right)\left(N_{0}<N\right)$ available, we now focus on how to extend the DCT model (Equations 1 and 2) to predict its future fluctuation at its next time point $\left(x(n)\left(n=N_{0}+\right.\right.$ 1)) or at its subsequent points in time: $x(n)\left(n=N_{0}+1, \ldots, N\right)$. However, as $N_{0}<N$, the principal problem is how to compute the DCT coefficients of $f(k)$ for forecast modeling (Equations 1 and 2) on the basis of the limited $N_{0}\left(N_{0}<N\right)$ observations.

Though only $N_{0}\left(N_{0}<N\right)$ observations available, our first thought may be to directly compute the DCT coefficients $f(k)$ by its original definition of Equation $1\left(f^{\prime}(k)=w(k) \sum_{n=1}^{N_{0}} x(n)\right.$ $\cos [\pi(2 n-1)(k-1) / 2 N])$, and then use its inverse transformation (Equation (2)) $\left(x^{\prime}(n)=\sum_{k=1}^{M} f^{\prime}(k) w(k) \cos [\pi(2 n-1)(k-1) /\right.$ $2 N])\left(M \leq N_{0}\right)$ to predict its future fluctuation. For instance, by direct computation of $f^{\prime}(k)$ and $x^{\prime}(k)$ using the original definetions of Equations 1 and 2, Figure 1 illustrates results of onestep-forecast $\left(N_{0}=N-1=23, M=11\right)$ for 24-hour AQI (measured in the U.S.A standard) fluctuations at the monitoring station of "Bantang" in Xiangtan of China on 2016-04 -23 (00:00-23:00) (GBMW, 2016). Where parameters of the forecast modeling are set as $N_{0}=N-1=23$ and $M=11$. That is, we use its $N_{0}=N-1=23$ previous hourly AQI observations: $x(n)\left(n=1,2, \ldots, N_{0}\right)$ to directly compute the $M$-term DCT coefficients $f^{\prime}(k)(k=1,2, \ldots, M)(M=11)$ by the original definition (Equation 1) (i.e., $f^{\prime}(k)=w(k) \sum_{n=1}^{N_{0}} x(n) \cos [\pi$ $(2 n-1)(k-1) / 2 N])$, and then we employ the DCT coefficients $f^{\prime}(k)$ to model and predict (Equation 2) (i.e., $x^{\prime}(n)=\sum_{k=1}^{M} f^{\prime}(k)$ $w(k) \cos [\pi(2 n-1)(k-1) / 2 N])$ the following one in the last hour of the day.

In Figure 1, it is shown that the forecasting result does not fit well with but deviates significantly from the AQI observation value.

Moreover, let us examine the DCT coefficients $f^{\prime}(k)$ by direct computation using the original definition (Equation 1)
( $N_{0}=23, N=24$ ) based on the previous $N_{0}=23$ hourly AQI observations. Figure 2 plots comparisons of the original 11term $(k=1,2, \ldots, M)(M=11)$ DCT coefficients $f(k)$ (Equation (1)) computed on the basis of the full 24-hourly AQI observations $\left(N_{0}=N=24\right)$ on 2016-4-23 (00:00-23:00) with the ones of $f^{\prime}(k)(k=1,2, \ldots, M)(M=11)$ approximated by direct computation using the original definition (Equation 1) $\left(N_{0}=23, N\right.$ $=24$ ) on the basis of its previous $N_{0}=23$ hourly AQI observations.

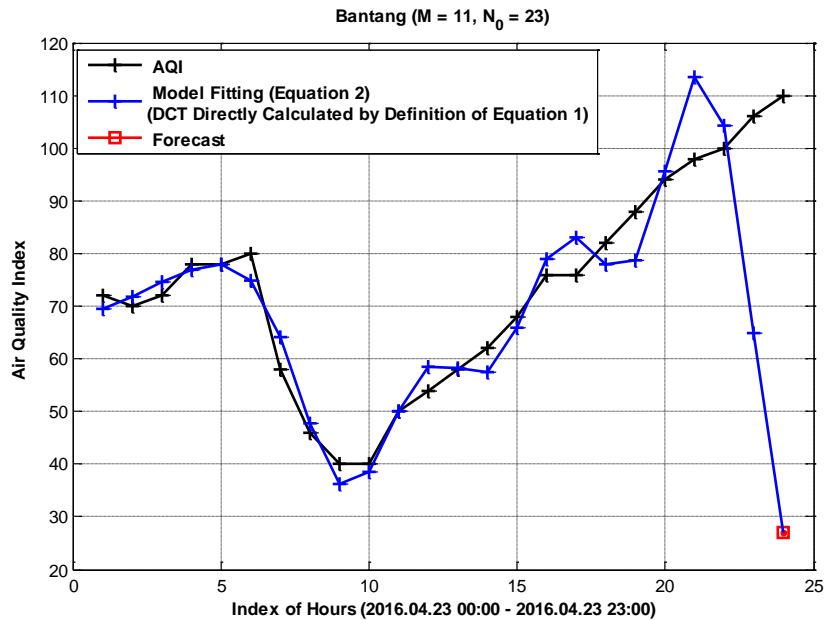

Figure 1. Plot of result of one-step-forecast $\left(N_{0}=N-1=23\right)$ for 24-hour AQI fluctuations at the monitoring station of "Bantang" in Xiangtan, China on 2016-04-23 (00:00 - 23:00) $(M=11)$ by direct computation using the DCT original definitions (Equations 1 and 2).

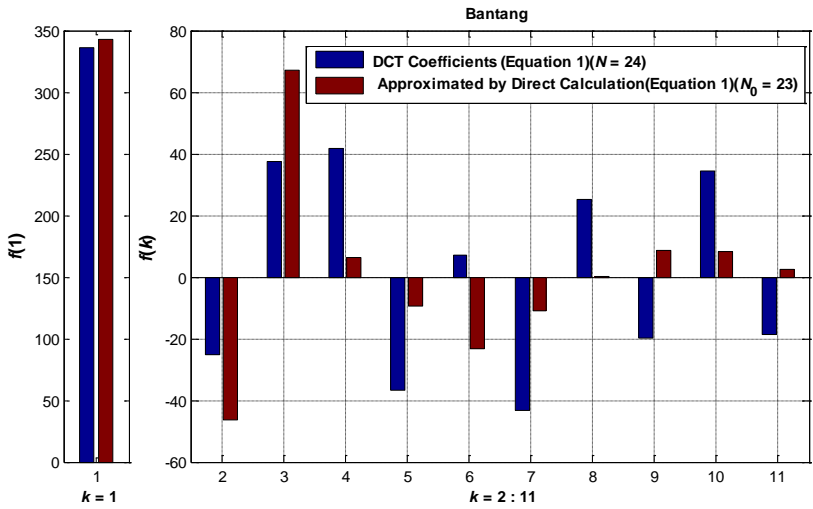

Figure 2. Comparisons of the 11-term $(M=11)$ DCT coefficients (Equation 1) calculated using full 24-hourly AQI observations $(N=24)$ on 2016-04-23 (00:00-23:00) with the ones approximated by the original definition (Equation 1) on the basis of its previous $N_{0}=23$ hourly AQI observations, respectively.

From Figure 2, it is also demonstrated that the 11-term $(M=11)$ DCT coefficients $f^{\prime}(k)$ approximated by direct computation using the original definition (Equation 1) $\left(N_{0}=23, N=\right.$ 24) on the basis of the previous $N_{0}=23$ hourly AQI observa- 
tions does not fit well with the original DCT coefficients $f(k)$ (Equation 1) $\left(N_{0}=N=24\right)$ which are calculated on the basis of the full 24-hourly AQI observations on 2016-4-23 (00:0023:00).

\subsection{The Proposed DCT-Based Least-Squares Predictive Model}

How to compute the DCT coefficients for forecast modeling on the basis of the limited number $N_{0}(<N)$ of hourly AQI observations, we now try to solve the problem in the leastsquares sense. An extended version of the DCT model (Equation 2) called the DCT-based least-squares predicttive model (Equation 3) is proposed as follows:

$$
x(n)=\sum_{k=1}^{M} f(k) w(k) \cos \left[\frac{\pi(2 n-1)(k-1)}{2 N}\right]
$$

where the called least-squares-optimum DCT coefficients $\hat{f}(k)$ $(k=1,2, \ldots, M)$ for the building DCT-based least-squares predictive model (Equation 3) will be determined on the basis of the finite $N_{0}\left(N_{0}<N\right)$ observations: $x(n)\left(n=1,2, \ldots, N_{0}\right)$ to yield $\hat{x}(n)$ (Equation 3$)$ most fitting the given $x(n)(n=1,2$, $\left.\ldots, N_{0}\right)\left(N_{0}<N\right)$ in the least-squares sense. Note that to effecttively get the $M$-term DCT coefficients $f(k)(k=1,2, \ldots, M)$ on the basis of the finite $N_{0}<N$ observations, normally, we set $M \leq N_{0}<N$.

The optimum solution in the least-squares sense of Equation 3 means that sum $E^{2}$ of squared deviations (residuals, errors) $e_{n}$ is to be one minimum:

$$
\begin{aligned}
E^{2} & =\sum_{n=1}^{N_{0}} e_{n}^{2}=\sum_{n=1}^{N_{0}}(x(n)-x(n))^{2} \\
& =\sum_{t=1}^{N_{0}}\left\{x(n)-\sum_{k=1}^{M} f(k) w(k) \cos \left[\frac{\pi(2 n-1)(k-1)}{2 N}\right]\right\}^{2}
\end{aligned}
$$

To minimize Equation 4 with respect to $\hat{f}(k)\left(\partial E^{2} / \partial \hat{f}(k)\right)=$ $0, k=1,2, \ldots, M)$, it results in:

$$
\begin{aligned}
& \sum_{n=1}^{N_{0}} w(k) \cos \left[\frac{\pi(2 n-1)(k-1)}{2 N}\right] \\
& \quad\left\{x(n)-\sum_{k=1}^{M} f(k) w(k) \cos \left[\frac{\pi(2 n-1)(k-1)}{2 N}\right]\right\}=0
\end{aligned}
$$
follows:

By denoting $c(m, n)=w(m) \cos [\pi(2 n-1)(k-1) / 2 N]$, it

$$
\begin{array}{ll}
\sum_{n=1}^{N_{0}} c(m, n) x(n) & \\
\quad=\sum_{n=1}^{N_{0}} c(m, n) \sum_{k=1}^{M} f(k) c(k, n) & \text { for } m=1,2, \ldots, M
\end{array}
$$

For brevity of mathematical expression, we define:

$$
\begin{aligned}
\boldsymbol{C}_{M \times N_{0}} & =\left[\begin{array}{cccc}
c(1,1) & c(1,2) & \ldots & c\left(1, N_{0}\right) \\
c(2,1) & c(2,2) & \ldots & c\left(2, N_{0}\right) \\
\ldots & \ldots & \ldots & \ldots \\
c(M, 1) & c(M, 2) & \ldots & c\left(M, N_{0}\right)
\end{array}\right], \\
\boldsymbol{x}_{N_{0} \times 1} & =\left[\begin{array}{c}
x(1) \\
x(2) \\
\ldots \\
x\left(N_{0}\right)
\end{array}\right], \boldsymbol{f}_{M \times 1}=\left[\begin{array}{c}
y(1) \\
y(2) \\
\ldots \\
y(M)
\end{array}\right]
\end{aligned}
$$

From Equations 6 and 7, it follows:

$$
\begin{aligned}
\boldsymbol{C}_{M \times N_{0}} \boldsymbol{x}_{N_{0} \times 1} & =\boldsymbol{C}_{M \times N_{0}}\left(\boldsymbol{f}_{1 \times M}^{T} \boldsymbol{C}_{M \times N_{0}}\right)^{T} \\
& =\boldsymbol{C}_{M \times N_{0}} \boldsymbol{C}_{N_{0} \times M}^{T} \boldsymbol{f}_{M \times 1}
\end{aligned}
$$

Note that for $m=1,2, \ldots, M$, we have $\sum_{n=1}^{N_{0}} c(m, n)$ $\sum_{k=1}^{M} \hat{f}(k) c(k, n)=\boldsymbol{C}_{M \times N_{0}}\left(\boldsymbol{f}_{1 \times M}^{T} \boldsymbol{C}_{M \times N_{0}}\right)^{T}$

Finally, from Equation 8, we can get the least-squareoptimum $M$-term DCT coefficients $\hat{f}(k)(k=1,2, \ldots, M)$ on the basis of the finite $N_{0}$ observations $x(n)\left(n=1,2, \ldots, N_{0}\right)$ $\left(N_{0}<N\right)$ by:

$$
\boldsymbol{f}_{M \times 1}=\left(\boldsymbol{C}_{M \times N_{0}} \boldsymbol{C}_{N_{0} \times M}^{T}\right)^{-1} \boldsymbol{C}_{M \times N_{0}} \boldsymbol{x}_{N_{0} \times 1}
$$

With using the least-squares-optimum DCT coefficients (Equation 9) to build the DCT-based least-squares predictive model (Equation 3), we can now predict future fluctuation of the AQI at its next time point $\hat{x}(n)\left(n=N_{0}+1\right)$ or at its subsequent points in time: $\hat{x}(n)\left(n=N_{0}+1, \ldots, N\right)$ by the DCTbased predictive model (Equation 3 ).

In summary, algorithm of the proposed DCT-based leastsquares predictive model (Equation 3) for forecasting hourly AQI fluctuation is composed of the following steps:

1. Put $N=24$ for describing hourly AQI fluctuation as 24 hours in each day.

2. Given finite $N_{0}\left(N_{0}<N\right)$ hourly AQI observations: $x(n)(n$ $\left.=1,2, \ldots, N_{0}\right)$, we are to forecast future fluctuation of the AQI at its next time point $\left.\hat{x}(n)\left(n=N_{0}+1\right)\right)$ or at its subsequent points in time: $\hat{x}(n)\left(n=N_{0}+1, \ldots, N\right)$.

3. To compute the least-squares-optimum $M$-term DCT coefficients: $\hat{f}(k)(k=1,2, \ldots, M)$ on the basis of the finite observations: $x(n)\left(n=1,2, \ldots, N_{0}\right)$ by $\boldsymbol{f}_{M \times 1}=\left(\boldsymbol{C}_{M \times N_{0}}\right.$ $\left.\boldsymbol{C}_{N_{0} \times M}^{T}\right)^{-1} \boldsymbol{C}_{M \times N_{0}} \boldsymbol{x}_{N_{0} \times 1}$ (Equations 6 to 9 ).

4. With the least-squares-optimum DCT coefficients $\hat{f}(k)(k$ $=1,2, \ldots, M)$ (Equation 9) available, future fluc- tuation of the hourly AQI at its next time point $\hat{x}(n)\left(n=N_{0}+1\right)$ or at its subsequent points in time $\left(n=N_{0}+1, \ldots, N\right)$ can be predicted by the DCT-based predictive model: $\hat{x}(n)=$ $\sum_{k=1}^{M} \hat{f}(k) w(k) \cos [\pi(2 n-1)(k-1) / 2 N]$ (Equation 3).

For instance, Figure 3 illustrates results of one-step-forecast $\left(N_{0}=N-1=23, M=12\right)$ for the 24-hour AQI (measured in the U.S.A standard) fluctuations at the monitoring station of 
"Bantang" in Xiangtan of China on 2016-04-23 (00:00-23:00) (GBMW, 2016) by using the proposed DCT-based leastsquares predictive model (Equations 3 and 9). Where parameters of the forecast modeling (Equations 3 and 9) are set as $N_{0}$ $=N-1=23$ and $M=11$. That is, we use its $N_{0}=N-1=23$ previous hourly AQI observations: $x(n)\left(n=1,2, \ldots, N_{0}\right)$ to compute the least-squares-optimum $M$-term DCT coefficients $\hat{f}(k)(k=1,2, \ldots, M)(M=11)$ (Equation 9) to model and forecast (Equation 3) the hourly AQI in the last hour of the day.

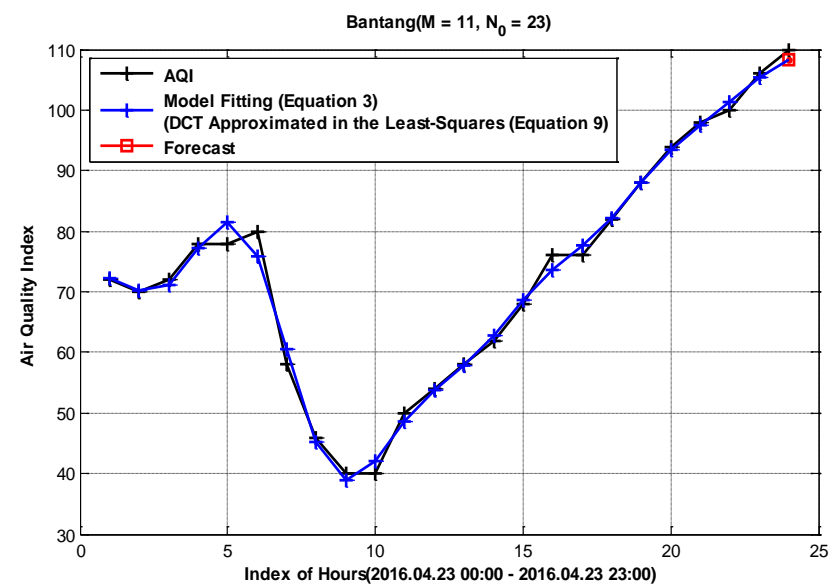

Figure 3. Plot of result of one-step-forecast $\left(N_{0}=N-1=23\right)$ for 24-hour AQI fluctuations at the monitoring station of "BanTang" in Xiangtan, China on 2016-04-23 (00:00-23:00) by the DCT-based least-squares predictive model (Equations 3 and 9$)$ by the setting of $(M=11)$.
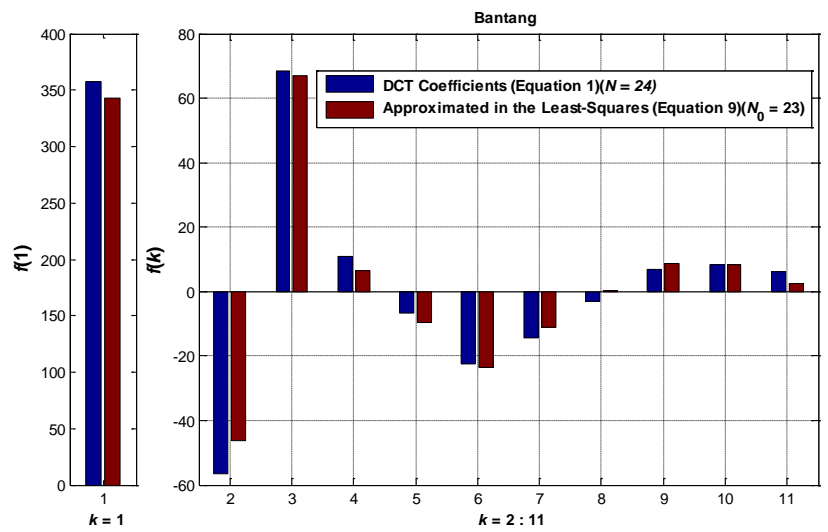

Figure 4. Comparisons of the 11-term $(M=11)$ DCT coefficients (Equation 1) calculated on the basis of the full 24hourly AQI observations $(N=24)$ on 2016-4-23 (00:00-23:00) and ones approximated in the least-squares sense (Equation 9) on the basis of its previous $N_{0}=23$ hourly AQI observations, respectively.

For the same prediction task, compared with the forecasting results (Figure 1) by direct computation using the original definitions (Equations 1 and 2), the prediction results (Figure 3 ) by the proposed DCT-based least-squares predictive model (Equations 3 and 9) agree well with the hourly AQI observa- tions.

Furthermore, Figure 4 plots comparisons of the leastsquares-optimum 11-term DCT coefficients $\hat{f}(k)(k=1,2, \ldots$, $M)(M=11)$ (Equation 9) $N_{0}=N-1=23$ approximated in the least-squares sense on the basis of the previous $N_{0}=23$ hourly AQI observations with the original ones $f(k)(k=1,2, \ldots, M)$ ( $M=11)$ (Equation 1) $\left(N_{0}=N=24\right)$ computed on the basis of the full 24-hourly AQI observations on 2016-4-23 (00:0023:00), respectively.

In comparison with the approximations of the DCT coefficients $f^{\prime}(k)(k=1,2, \ldots, M)(M=11)$ (Figure 2) by direct computation based on the previous $N_{0}=23$ hourly AQI observations using the original definition, it is shown (Figure 4) that the called least-squares-optimum 11-term DCT coefficients $\hat{f}(k)(k=1,2, \ldots, M)(M=11)$ (Equation 9) approximated in the least-squares sense (Equation 9) on the basis of the previous $N_{0}=23$ hourly AQI observations fit well with the original DCT $f(k)(k=1,2, \ldots, M)(M=11)$ (Equation 1) computed on the basis of the full 24-hourly AQI observations $(N=24)$ on 2016-4-23 (00:00-23:00).

The above results also prove feasibility of the proposed DCT-based least-squares predictive model (Equations 3 and 9). Meanwhile, the DCT coefficients displayed in Figure 4 also confirms the strong "energy compaction" property of DCT (Zheng et al., 2011). That is, value of the DCT coefficient $f(k)(k=1)$ (i.e., the DC coefficient) is much larger than values of the other DCT coefficients $f(k)(k=2, \ldots, 12)$ (i.e., the AC coefficients) while the lower AC coefficients $f(k)$ (such as, $k=2,3$ in Figure 4) are also much larger than the higher AC coefficients $f(k)(k \geq 4)$.

Furthermore, for the same prediction instance, some illustrations of different settings ( $M=12,5,7,20$, and 22) of parameter $M$ ( $M$-term least-squares-optimum DCT coefficients) for the proposed the DCT-based least-squares predictive model are presented in Figures 5 to 9, respectively.

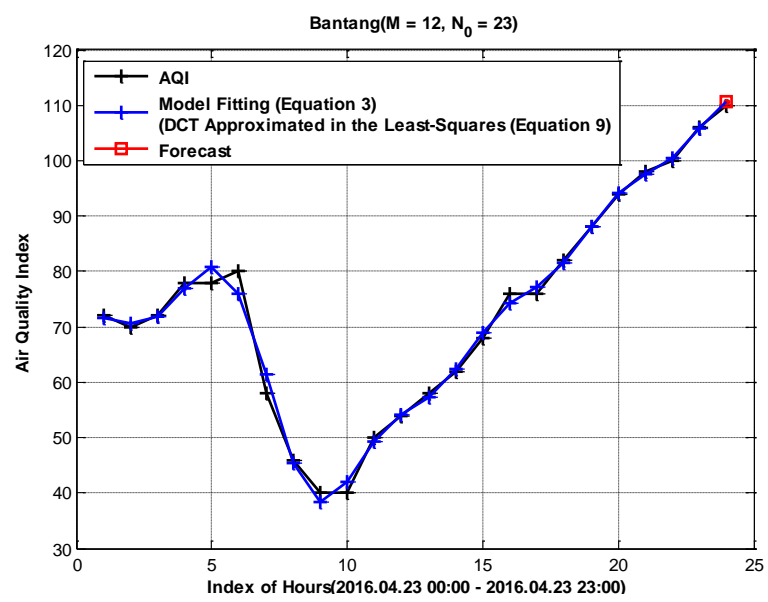

Figure 5. Plot of result of one-step-forecast $\left(N_{0}=N-1=23\right)$ for 24-hour AQI fluctuations at the monitoring station of "Bantang" in Xiangtan, China on 2016-04-23 (00:00- 23:00) by the DCT-based least-squares predictive model (Equations 3 and 9) with the setting of $(M=12)$. 


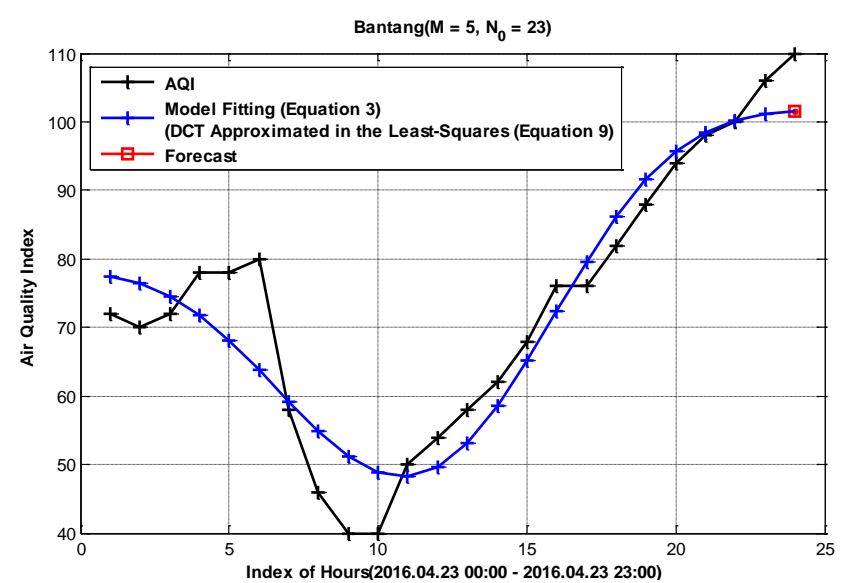

Figure 6. Plot of result of one-step-forecast $\left(N_{0}=N-1=23\right)$ for 24-hour AQI fluctuations at the monitoring station of "Bantang" in Xiangtan, China on 2016-04-23 (00:00-23:00) by the DCT-based least-squares predictive model (Equations 3 and 9) with the setting of $(M=5)$.

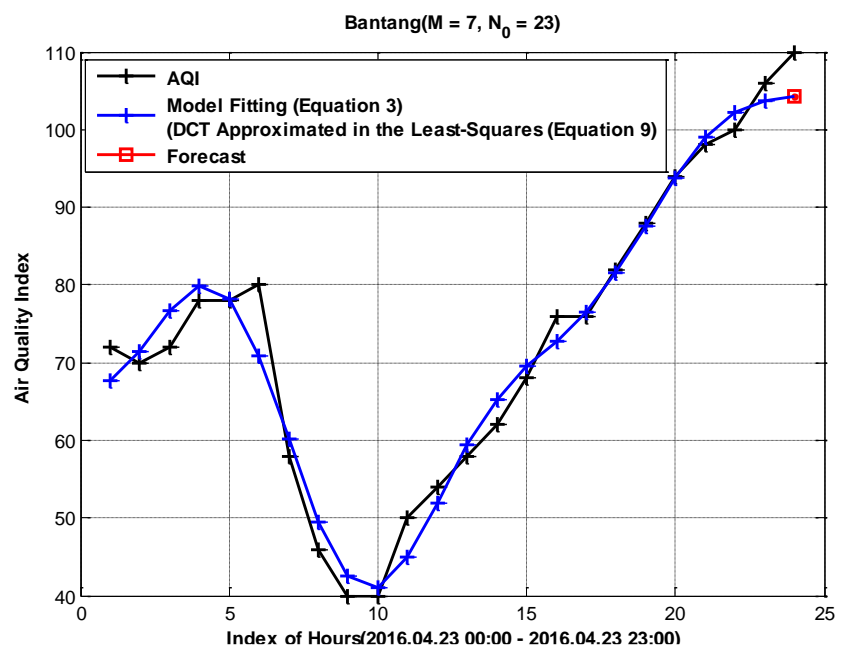

Figure 7. Plot of result of one-step-forecast $\left(N_{0}=N-1=23\right)$ for 24-hour AQI fluctuations at the monitoring station of "Bantang" in Xiangtan, China on 2016-04-23 (00:00-23:00) by the DCT-based least-squares predictive model (Equations 3 and 9) with the setting of $(M=7)$.

From Figure 3 and Figures 5 to 9, it is shown that the hourly AQI fluctuations can be well predicted by the proposed DCT-based least-squares predictive model (Equation 3) with using about 12-term $(M=11,12)$ least-squares-optimum DCT coefficients. One explanation for the other settings of $(M=5$, $7)$ and $(M=20,22)$ may be that the former ones of $(M=5,7)$ and the latter ones of $(M=20,22)$ seems to be "under-fitting" and "over-fitting", respectively.

\section{Case Study}

Firstly, the proposed DCT-based least-squares predictive model (Equation 3) is applied to forecast hourly AQI (mea- sured in the U.S.A standard (GBMW, 2016)) fluctuations at five monitoring stations during six random 48-hour periods from 2016-4-22 9:00 to 2016-4-24 8:00, from 2016-4-22 $17: 00$ to $2016-4-2416: 00$, and from $2016-4-23$ 8:00 to 20164-25 7:00, 2016-5-7 4:00 to 2016-5-12 3:00, 2016-5-25 5:00 to 2016-5-31 4:00 and 2016-6-1 5:00 to 2016-6-8 4:00, respectively. Locations of the five monitoring stations ("Yuetang", "Central", "Bantang”, "Jianglu", "Keda") in Xiangtan of China are illustrated in Figure 10.

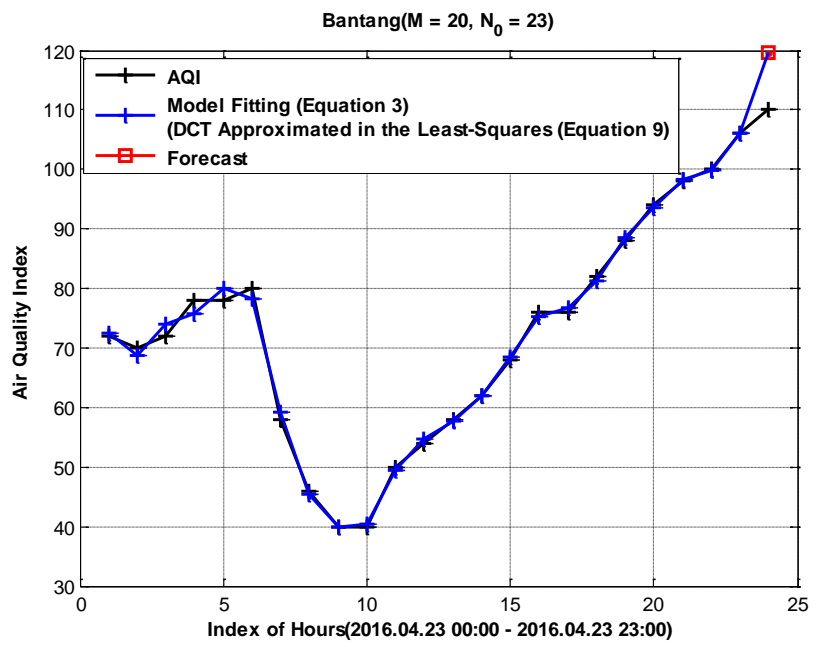

Figure 8. Plot of result of one-step-forecast $\left(N_{0}=N-1=23\right)$ for 24-hour AQI fluctuations at the monitoring station of "Bantang" in Xiangtan, China on 2016-04-23 (00:00- 23:00) by the DCT-based least-squares predictive model (Equations 3 and 9) with the setting of $(M=20)$.

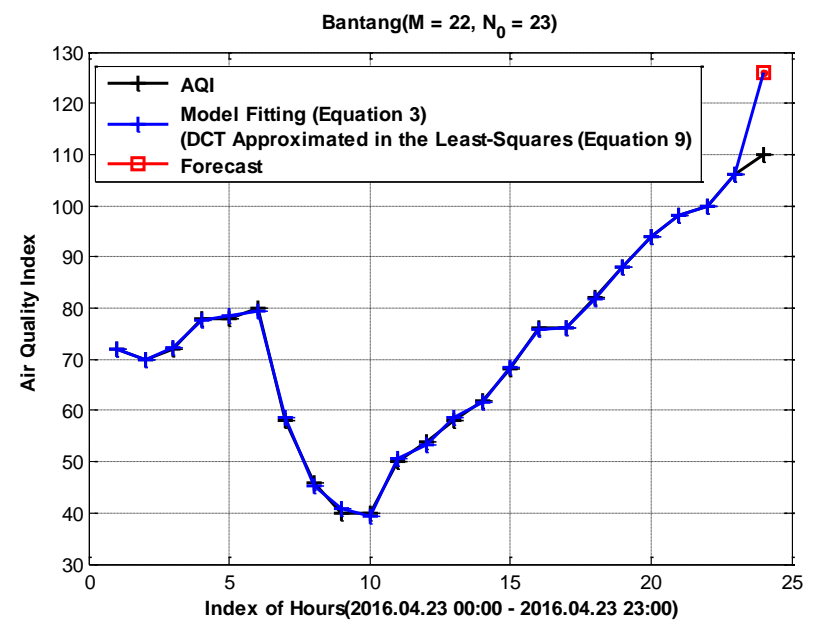

Figure 9. Plot of result of one-step-forecast $\left(N_{0}=N-1=23\right)$ for 24-hour AQI fluctuations at the monitoring station of "Bantang" in Xiangtan, China on 2016-04-23 (00:00-23:00) $(M=22)$ by the DCT-based least-squares predictive model (Equations 3 and 9$)$ by the setting of $(M=22)$.

Hourly AQI observations of the five monitoring stations were published by the called Green-Breath monitoring web- 
site of "www.pm25.com" in China (GBMW, 2016). Based on $N_{0}=23$ hourly AQI observations and by using two different $(M=12,11)$ least-squares-optimum DCT coefficients (Equation 9) in forecast modeling, prediction results of the proposed DCT-based model (Equation 3) $(N=24)$ are presented as follows.

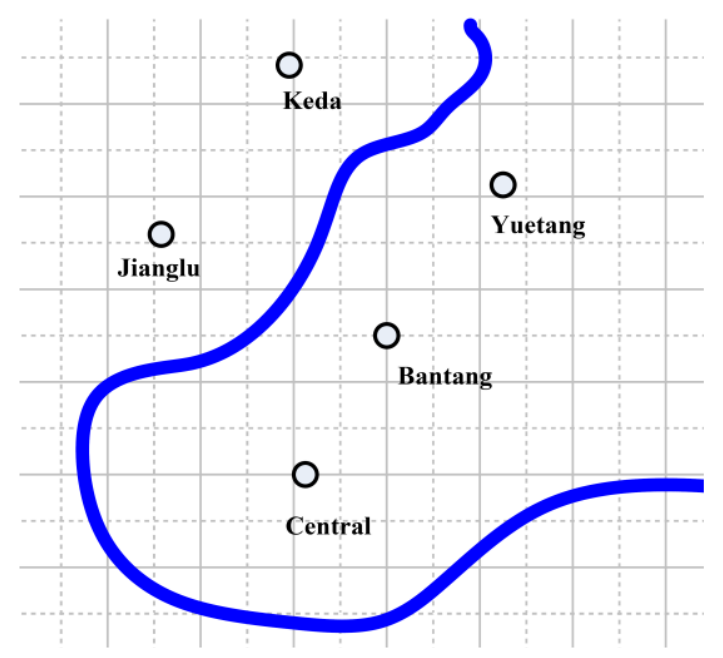

Figure 10. Illustration of locations of the 5 monitoring stations ("Yuetang", "Central", "Bantang", "Jianglu", "Keda") in Xiangtan of China.

With using 12-term least-squares-optimum DCT coefficients $(M=12)$ (Equations 6 and 9) in forecast modeling (Equation 3), Figure S1 plot results of 25 one-step-forecast $\left(N_{0}=N-1=23\right)$ for 48-hour AQI fluctuations at the monitoring station of "Yuetang" in Xiangtan of China from 20164-22 9:00 to 2016-4-24 8:00. Where parameters of the proposed DCF-based predictive model (Equations 3,6 and 9) are set as $N_{0}=N-1=23$ and $M=12$. That is, at each step, we use its previous $N_{0}=23$ hourly AQI observations to compute the least-squares-optimum $M$-term DCT coefficients $\hat{f}(k)(k=$ $1,2, \ldots, M)(M=12)$ (Equations 6 and 9) to model and predict (Equation 3) its following one, and then there are 25 one-step -forecast from 2016-4-22 9:00 to 2016-4-24 8:00.

Under the same settings $\left(N_{0}=23, N=24 ; M=12\right)$ in forecast modeling (Equations 3, 6 and 9), results of 25 onestep-forecast for the 48-hour AQI fluctuations at the other 4 monitoring stations ("Central", "Bantang", "Jianglu", "Keda") in Xiangtan, China from 2016-4-22 9:00 to 2016-4-24 8:00 are listed in Tables 1 and S1, respectively.

In the similar way and under the same settings of $\left(N_{0}=\right.$ $23, N=24 ; M=12$ ) for the proposed DCT-based forecast modeling (Equations 3, 6 and 9), results of 25 one-step-forecast for hourly AQI fluctuations at the five monitoring stations ("Yuetang" (demonstrated in Figure S2), "Central", "Bantang", "Jianglu", "Keda") in Xiangtan of China during the random 48-hour period from 2016-4-22 17:00 to 2016-4-24 16:00 are listed in Tables 1 and S1, respectively.

Similarly, with using 11-term least-squares-optimum DCT coefficients $(M=11)$ (Equations 6 and 9) for the proposed DCT-based forecast modeling (Equation 3) $\left(N_{0}=23, N=24\right)$, results of 25 one-step-forecast for hourly AQI fluctuations at the five monitoring stations ("Yuetang" (demonstrated in Figure S3), "Central", "Bantang", "Jianglu", "Keda") in Xiangtan of China during the random 48-hour period from 2016-4-23 7:00 to 2016-4-25 6:00 are also listed in Tables 1 and S1, respectively.

Moreover, in the similar way, forecasting results using the proposed DCF-based least-squares predictive model (Equations 3 and 9) for 3 other random long periods (i.e., one random 120-hour period from 2016-5-7 4:00 to 2016-5-12 3:00, one random 144-hour period from 2016-5-25 5:00 to 2016-531 4:00 and one random 168-hour period form 2016-6-1 5:00 to 2016-6-8 4:00) are presented as follows.

Under the settings of $\left(N_{0}=23, N=24 ; M=12\right)$ for the proposed DCT-based forecast modeling (Equations 3 and 9), results of 97 one-step-forecast for hourly AQI fluctuations at the five monitoring stations ("Yuetang" (demonstrated in Figure S4), "Central", "Bantang", "Jianglu", "Keda") in Xiangtan of China during the random 120-hour period from 2016-5-7 4:00 to 2016-5-12 3:00 are also listed in Tables 1 and $S 1$, respectively.

Table 1. Percents (\%) of RMSE-to-Mean in Predicting the Hourly AQI Fluctuations by Using the Proposed DCT-Based Least-Squares Predictive Model

\begin{tabular}{|c|c|c|c|c|c|}
\hline $\begin{array}{l}\text { Monitoring Station }\left(N_{0}=23, N=24\right) \\
(M=11,12)\end{array}$ & Central (\%) & Bantang (\%) & Jianglu (\%) & Keda $(\%)$ & Yuetang (\%) \\
\hline $\begin{array}{l}25 \text { one-step-forecast } \\
\text { (2016-4-22 9:00 to 2016-4-24 8:00) }\end{array}$ & 8.872 & 6.311 & 5.929 & 6.515 & 3.760 \\
\hline $\begin{array}{l}25 \text { one-step-forecast } \\
\text { (2016-4-22 17:00 to } 2016-4-24 \text { 16:00) }\end{array}$ & 8.329 & 6.303 & 5.310 & 5.896 & 3.481 \\
\hline $\begin{array}{l}25 \text { one-step-forecast } \\
\text { (2016-4-23 7:00 to } 2016-4-25 \text { 6:00) }\end{array}$ & 7.636 & 5.810 & 4.708 & 5.076 & 3.258 \\
\hline $\begin{array}{l}97 \text { one-step-forecast } \\
\text { (2016-5-7 4:00 to } 2016-5-12 \text { 3:00) }\end{array}$ & 8.677 & 8.239 & 6.517 & 5.012 & 4.622 \\
\hline $\begin{array}{l}121 \text { one-step-forecast } \\
\text { (2016-5-25 5:00 to } 2016-5-31 \text { 4:00) }\end{array}$ & 10.724 & 8.860 & 7.108 & 6.393 & 4.219 \\
\hline $\begin{array}{l}145 \text { one-step-forecast } \\
\text { (2016-6-1 5:00 to 2016-6-8 4:00) }\end{array}$ & 12.832 & 9.617 & 9.099 & 7.373 & 6.330 \\
\hline
\end{tabular}


Table 2. Percents (\%) of RMSE-to-Mean in Forecasting the Hourly AQI Fluctuations by Using the BP-ANN Model

\begin{tabular}{|c|c|c|c|c|c|}
\hline Monitoring Station & Central (\%) & Bantang (\%) & Jianglu (\%) & Keda $(\%)$ & Yuetang (\%) \\
\hline $\begin{array}{l}25 \text { one-step-forecast } \\
(2016-4-22 \text { 9:00 to } 2016-4-24 \text { 8:00) }\end{array}$ & 21.753 & 25.230 & 21.390 & 27.311 & 12.528 \\
\hline $\begin{array}{l}25 \text { one-step-forecast } \\
\text { (2016-4-22 17:00 to 2016-4-24 16:00) }\end{array}$ & 15.538 & 16.009 & 12.932 & 14.007 & 17.335 \\
\hline $\begin{array}{l}25 \text { one-step-forecast } \\
\text { (2016-4-23 7:00 to } 2016-4-25 \text { 6:00) }\end{array}$ & 16.253 & 16.505 & 12.483 & 12.082 & 14.371 \\
\hline $\begin{array}{l}97 \text { one-step-forecast } \\
\text { (2016-5-7 4:00 to } 2016-5-12 \text { 3:00) }\end{array}$ & 39.253 & 37.689 & 34.555 & 34.673 & 32.573 \\
\hline $\begin{array}{l}121 \text { one-step-forecast } \\
(2016-5-25 \text { 5:00 to } 2016-5-314: 00)\end{array}$ & 39.206 & 32.650 & 29.606 & 26.920 & 17.953 \\
\hline $\begin{array}{l}145 \text { one-step-forecast } \\
\text { (2016-6-1 5:00 to 2016-6-8 4:00) }\end{array}$ & 37.367 & 37.786 & 35.918 & 31.520 & 28.699 \\
\hline
\end{tabular}

Under the settings of $\left(N_{0}=23, N=24 ; M=11\right)$ for the proposed DCT-based forecast modeling (Equations 3 and 9), results of 121 one-step-forecast for hourly AQI fluctuations at the five monitoring stations ("Yuetang" (demonstrated in Figure S3), "Central", "Bantang", "Jianglu", "Keda") in Xiangtan of China during the random 144-hour period from 2016-5-25 5:00 to 2016-5-31 4:00 are listed in Tables 1 and S1, respecttively. And in the same way, results of 145 one-step-forecast $\left(N_{0}=23, N=24 ; M=11\right)$ for hourly AQI fluctuations at the five monitoring stations ("Yuetang" (demonstrated in Figure S4), "Central", "Bantang", "Jianglu", "Keda") in Xiangtan of China during the random 168-hour period from 2016-6-1 5:00 to 2016-6-8 4:00 are also listed in Tables 1 and S1, respecttively.

The experimental results (such as Figures S1 to S4) show that the forecasting results agree well with the hourly AQI fluctuations at the monitoring stations.

\section{Result Analyses and Discussions}

Performance analysis of the proposed DCT-based leastsquares predictive model with the classical BP-ANN ("Feedforward Backpropagation" ANN) model, the Fourier-seriesbased least-squares predictive model (Yang, 2013, 2014) and the ARIMA model for the same prediction tasks, limitation and advantage of the proposed DCT-based model are presented in this section.

\subsection{RMSE-to-Mean-Based Analysis}

For the forecasting results of hourly AQI fluctuations at the five monitoring stations in Xiangtan of China during the 6 random periods, Table 1 lists percentages (\%) of RMSE-tomean by using the proposed DCT-based least-squares predictive model (Equations 3 and 9) $\left(N=24, N_{0}=N-1\right)(M=11$ and 12), respectively. Where the $R M S E=\sqrt{\sum_{n=1}^{R}(\widehat{x}(n)-x(n))^{2} / R}$ $(\hat{x}(n)$ is the forecast value for $x(n)$ at time point of $n)$ refers to "root-mean-square-error". It is also known as the standard deviation of prediction error. Normalizing the RMSE can facilitate the comparison between datasets or models with different scales. One common choice is to use the mean of the mea- sured data to normalize the RMSE, i.e., the RMSE-to-mean (the RMSE divided by mean of $x(n)$ ).

Various factors may affect hourly AQI fluctuation, such as the temperature, light intensity, wind speed, humidity and so on. These parameters may be used in predicting the AQI fluctuation. From the viewpoint of time-series analysis or data-driven modeling, hourly AQI fluctuation implicating all its influential factors is also one time-series. Thus all the factors influencing hourly AQI fluctuation are implicitly enclosed in its time-sequence. Then only the time-series data of hourly AQI is used in the proposed DCT-based predictive model (Equation 3) while other parameters are not directly involved in the proposed model. Hence the most widely used BP-ANN model can be taken as one of the most comparable competitive models. Performance comparisons of the proposed DCT-based least-squares predictive model (Equation 3) with the classical BP-ANN model are presented as follows.

Many rule-of-thumb approaches for determining the proper number of neurons in the hidden layer of one ANN model have been reported, such as, the 3 simple rules (Heaton, 2005) (i.e., the number of hidden neurons may be (1) within the range of the sizes of the input layer and the output layer; (2) two-thirds of the input layer size plus the output layer size; (3) less than twice of the input layer size). Actually, the final selection of an ANN structure may come down to "trial-anderror" (Heaton, 2005). For the same prediction tasks and under the same condition (to use the given $N_{0}$ previous observations for training), the network structure of the BP-ANN model is chosen as "12-26-1". The network structure of "1226-1" denotes that there are 12 nodes in the input layer, 26 nodes in the hidden layer, and one node in the output layer. The BP-ANN model uses the log-sigmoid activation function in the hidden layer. In terms of percentages (\%) of RMSE-tomean, Tables 2 and 3 list performance comparisons of the proposed DCT-based least-squares model with the BP-ANN model for the same prediction tasks, respectively.

The ANOVA ("analysis of variance") is a parametric procedure usually employed to identify whether or not significant differences exist between two or more group means. Normally, the small $p$-value indicates that differences between the group means are highly significant (such as in Tables 4 and S3). 
Table 3. Comparisons of Performance in Terms of Average RMSE-to-Mean for Forecasting the Hourly AQI Fluctuations by Using the Proposed DCT-Based Model and the BP-ANN Model

\begin{tabular}{lll}
\hline Time period & The Proposed DCT-based Model (\%) & The BP-ANN Model (\%) \\
\hline $\begin{array}{l}25 \text { one-step-forecast } \\
\text { (2016-4-22 9:00 to 2016-4-24 8:00) }\end{array}$ & 6.277 & 21.642 \\
$\begin{array}{l}25 \text { one-step-forecast } \\
\text { (2016-4-22 17:00 to 2016-4-24 16:00) }\end{array}$ & 5.864 & 15.164 \\
25 one-step-forecast & 5.298 & 14.339 \\
(2016-4-23 7:00 to 2016-4-25 6:00) & & \\
97 one-step-forecast & 6.613 & 35.749 \\
(2016-5-7 4:00 to 2016-5-12 3:00) & 7.461 & 29.267 \\
$\begin{array}{l}\text { 121 one-step-forecast } \\
\text { (2016-5-25 5:00 to 2016-5-31 4:00) }\end{array}$ & 9.050 & 34.258 \\
145 one-step-forecast \\
$\begin{array}{l}2016-6-1 \text { 5:00 to 2016-6-8 4:00) } \\
\text { Average RMSE-to-Mean } \\
\text { Average Predictive Accuracy }\end{array}$
\end{tabular}

Table 4. Result of the ANOVA Statistical Test for Performances between the Proposed DCT-Based Model and the BP-ANN Model for the Same Forecasting Tasks

\begin{tabular}{llllll}
\hline Source of Variation & Sum of Squares & Degree of freedom & Mean Squares & F & P-value \\
\hline Between & 0.10057 & 1 & 0.10057 & 22.347 & $8.075 \times 10^{-4}$ \\
Within & 0.04500 & 10 & 0.0045003 & & \\
Total & 0.14557 & 11 & & & \\
\hline
\end{tabular}

In terms of the average RMSE-to-mean (Table 3), result of the ANOVA statistical test for performances between the proposed DCT-based least-squares predictive model and the BPANN model for the same prediction tasks is listed in Table 4.

From Tables 3 and 4 , the reported small $p$-values (significant level) $8.075 \times 10^{-4}<0.1$ further confirms that the proposed DCT-based least-squares predictive model outperforms the BP-ANN for the same tasks of forecasting the AQI fluctuations.

The DCT is a Fourier-related transform. So the proposed DCT-based least-squares predictive model and the Fourierseries-based least-squares predictive model (Yang, 2013, 2014) are Fourier-related extended predictive models. Compared with the latter model (Yang, 2013, 2014), the proposed DCT-based model is somewhat concise because mathematical formulations of the proposed model are somewhat more concise than that of the latter model. While physics meaning of the latter model (i.e., the Fourier-series coefficients and harmonics) (Yang, 2013, 2014) is more clear and definite than that of the proposed DCT-based least-squares predictive model (the transform (DCT) coefficients, which may also be referred to as the DC Coefficient $(k=1)$ and the AC Coefficients $(k=2, \ldots, N)$ (Sayood and Borkenhagen, 1991). In terms of percentages (\%) of RMSE-to-mean for the same forecasting tasks, Table S2 and S3 list performance comparisons of the proposed DCT-based least-squares predictive model with the Fourierseries-based least-squares predictive model (Yang, 2013, 2014) (with using 6-terms harmonics), respectively. Then in terms of the average RMSE-to-mean (Table S3), result of the ANOVA statistical test for performances between the proposed DCT- based least-squares predictive model and the Fourier-series-based least-squares predicttive model for the same prediction tasks is listed in Table S4.

From Tables 1 and $\mathrm{S} 2$ to $\mathrm{S} 4$, the reported small $p$-values (significant level) $6.861 \times 10^{-4}<0.1$ further confirms that the proposed DCT-based least-squares predictive model perform better than the Fourier-series-based least-squares predictive model for the same tasks of forecasting the AQI fluctuations.

The ARIMA model is also one widely-used model for time-series forecasting. In terms of percentages (\%) of RMSEto-mean for the same forecasting tasks, Tables S5 to S7 list performance comparisons of the proposed DCT-based leastsquares predictive model with the ARIMA model, respecttively.

From the comparison results in Tables S5 to S6 as well as the high $p$-values (significant level) of $0.631>0.1$ in Table S6, it follows that the proposed DCT-based least-squares predictive model perform slightly better than or comparable to the ARIMA model for the same prediction tasks.

\subsection{Correlation Coefficient-based Analysis}

Moreover, another measure index called the correlation coefficient (Pearson correlation coefficient) is employed to evaluate the prediction results by using the different models. The correlation coefficients of the different models for the same forecasting tasks are listed in Tables S1 and S8 to S10, respectively.

From the correlation coefficients of the different models listed in Tables S1 and S8 to S10, it is also shown that for the 
same tasks of forecasting the AQI fluctuations, the proposed DCT-based least-squares model performs better than the other two models (the BP-ANN model and the Fourier-series-based least-squares model) and performs comparable to the ARIMA model.

\subsection{Advantage of the Proposed DCT-Based Forecast Model}

In summary, the proposed method yields one good result of average $93.24 \%$ predictive accuracy in the forecast experiments at five monitoring stations in Xiangtan of China. On the basis of evaluation indices of the "RMSE-to-mean" and the correlation coefficients (Tables 1 to 4 and $\mathrm{S} 1$ to $\mathrm{S} 10$ ), it is demonstrated that for the same tasks of forecasting the AQI fluctuations, the proposed DCT-based least-squares model performs better than the other two models (the BP-ANN model and the Fourier-series-based least-squares model), and performs slightly better than or comparable to the ARIMA model.

From time-series analysis or data-driven modeling perspective, hourly AQI fluctuation implicating all its influential factors is also one time-series. It means that all the factors influencing the AQI fluctuation are implicitly enclosed in its time-sequence. Then only the time-series data of hourly AQI is used in the proposed DCT-based forecast model while other parameters are not directly involved. From the above analysis and discussions, we may see that concise and well-formed may be one major advantage of the proposed DC-based leastsquares predictive model for hourly AQI prediction.

Nevertheless, it seems to be obvious that there currently does not exist one method or model can meet all requirements for all specific problem instances. Limitations of the proposed DCT-based forecast model are listed below.

\subsection{Limitation of the Proposed DCT-Based Forecast Model}

The meteorological factors can largely influence particle pollution in the air. In this study, the DCT-based least-squares predictive model is proposed for hourly AQI forecasting from the perspective of time-series or data-driven modeling. The perspective indicates that fluctuation of daily 24-hour air quality that may be influenced by various factors is also one timeseries. Thus fluctuations in its time-series implicitly reflect various factors affecting the air quality. Then only the timeseries data of hourly AQI is used in the proposed DCT-based least-squares predictive model (Equation 3). Usually, the time interval $\Delta t$ for hourly AQI observation is one hour $(\Delta t=1$ hour). However, the weather may change quickly in some areas or in some times such as in winters. In these cases, the sudden or quick changes of the meteorological factors cannot be quickly recorded or reflected in previous observations of hourly AQI time-series, so that data-driven or time-seriesbased predictive models are usually incapable to respond to the sudden or quick changes. One possible solution may be to take more samples with smaller sampling interval of time $\Delta t$ for the AQI observations.
Another consideration of the proposed method is that the selection of the least-squares-optimum DCT coefficients in forecast modeling may be somewhat different for other specific case. And the settings $\left(M=11,12 ; N_{0}=N-1=23\right)$ in the experiments may benefit other applications. In general, the prediction accuracy will be better or higher when more previous hourly AQI observations are available or given to compute the least-squares-optimum DCT coefficients in forecast modeling. For instance, in the cases to take much more samples with smaller sampling interval of time $\Delta t$ for the AQI observations. Such as, when setting $\Delta t=0.5$ hour, we put $N=48$ for describing the half-hourly AQI. Accordingly, in the similar way, we may use about 24-term $(M=23 \sim 24)$ least-squaresoptimum DCT coefficients to model and predict (Equations 3 and 9) the half-hourly AQI. Nevertheless, its further investigation should be emphasized in our future study.

\section{Conclusions}

Human daily activities and meteorological factors may largely influence particle pollution in the air. From the viewpoint of time-series analysis or data-driven modeling, hourly AQI fluctuation, which may be influenced by various factors, is also one time-series. Thus all the factors influencing hourly AQI fluctuation are implicitly enclosed in its time-series. Fourier-based analysis technology is a powerful tool for timeseries analysis while DCT is also a Fourier-related transform. However, the conventional Fourier-based technology cannot be directly used for forecasting. In this study, a DCT-based predictive model extended in the least-squares sense is proposed for hourly AQI fluctuation forecasting. Contributions of this study are:

The DCT-based least-squares predictive model (Equation 3 ) is proposed for hourly AQI fluctuation forecasting from the perspective of time-series analysis or data-driven modeling. The proposed predictive model (Equation 3) is implemented in conjunction with the least-squares method to compute its least-squares-optimum DCT coefficients (Equations 6 to 9) on the basis of finite hourly AQI observations. Then the called least-squares-optimum DCT coefficients (Equations 6 to 9) are used to model and predict (Equation 3) future fluctuation of the AQI.

Experiments and result analysis indicate potentiality of the proposed method. The experiments show that the proposed method yields one satisfying result of average $93.24 \%$ predictive accuracy in the forecast experiments at five monitoring stations in Xiangtan of China. Performance comparesons of the proposed DCT-based least-squares predictive model with the classical BP-ANN model, the Fourier-series-based least-squares predictive model (Yang, 2013, 2014) and the ARIMA model indicate that for the same tasks of forecasting the hourly AQI fluctuations, the proposed DCT-based predicttive model outperforms the other two competitive models (the BP-ANN model and the Fourier-series-based least-squares predictive model) and performs slightly better than or comparable to the ARIMA model.

It is indicated that the hourly AQI fluctuations can be 
well predicted by the proposed DCT-based least-squares predictive model (Equation 3) with using about 12-term leastsquares-optimum DCT coefficients. For the daily 24-hour AQI fluctuation prediction, we often put $N=24$. Normally, different N0-length $\left(N_{0}<N=24\right)$ data used in forecast modeling yield different forecasting results. In general, the prediction accuracy will be better or higher when more previous hourly AQI observations are available or given to compute the least-squares-optimum DCT coefficients used for forecast modeling. Thus, the parameter settings may be somewhat different for other specific case. And our settings $\left(M=11,12 ; N_{0}=23\right)$ in the experiments may benefit other applications. Usually, the time interval $\Delta t$ for hourly AQI observation is one hour $(\Delta t$ $=1$ hour). However, the weather may change quickly in some areas or in some times. In these cases, the sudden or quick changes of the meteorological factors cannot be quickly recorded or reflected in previous observations of hourly AQI time-series, so that data-driven or time-series-based predictive models are usually incapable to respond to the sudden or quick changes. In the cases, one possible solution may be to take more samples with smaller sampling interval of time $\Delta t$ for the AQI observations. For instance, when setting $\Delta t=0.5$ hour, we put $N=48$ for describing the half-hourly AQI. Accordingly, in the similar way, we may use about 24-term ( $M=23$ and 24, $N_{0}=N-1$ ) least-squares-optimum DCT coefficients to model and predict (Equations 3 and 9) the halfhourly AQI.

Hourly AQI fluctuation implicating all its influential factors is also one time-series. Since all the factors influencing the AQI fluctuation are implicitly enclosed in its time-sequence, only the time-series data of hourly AQI is used in the proposed DCT-based least-squares predictive model while other parameters are not directly involved. Thus concise and wellformed may be one major advantage of the proposed DCTbased least-squares predictive model for hourly AQI forecasting. However, we cannot expect that currently there exists one algorithm or model can outperform all others on all instances. Hybrid modeling by combing one technique with other ones will be one promising direction (Yu et al., 2014). Thus, further investigation and improvement of the proposed DCTbased least-squares predictive model should be subject to our future study.

Acknowledgments. The research was supported by SRFHPSTD (2013GK3090) ("Scientific Research Fund of Hunan Provincial Science and Technology Department, China"). The author would like to extend his thanks to the Green-Breath Monitoring Website (http:// www.pm25.com/) for the data sharing in the Internet. The author would also like to extend his appreciation to the editor(s) and anonymous reviewers for their valuable comments and suggestions.

\section{References}

Benis, K.Z., Fatehifar, E., Shafiei, S., Nahr, F.K., and Purfarhadi, Y. (2013). Design of a sensitive air quality monitoring network using an integrated optimization approach. Stochastic Environ. Res. Risk Assess., 30(3), 779-793. https://doi.org/10.1007/s00477-015-1189-z

Briggs, D. (2003). Environmental pollution and the global burden of disease. Br. Med. Bull., 68(1), 1-24. https://doi.org/10.1093/bmb/ $\operatorname{ldg} 019$

Chatfield, C. (2004). The Analysis of Time Series, Chapman \& Hall/CRC, New York.

Chatterjee, S., Hadi, A., and Price, B. (2000). Simple Linear Regression. Ch. 2 in Regression Analysis by Example (3rd edition). New York: Wiley, pp. 21-50.

Chen, R.J., Wang, X., Meng, X., Hua, J., Zhou, Z.J., Chen, B.H., and Kan, H.D. (2013). Communicating air pollution-related health risks to the public: An application of the Air Quality Health Index in Shanghai, China. Environ. Int., 51, 168-173. https://doi.org/10. 1016/j.envint.2012.11.008

Cheng, W.L., Zhang, J.F., Lyons, T.J., Paid, J.L., and Chang, S.H. (2007). Comparison of the Revised Air Quality Index with the PSI and AQI indices. Sci. Total Environ., 382(2-3), 191-198. https:// doi.org/10.1016/j.scitotenv.2007.04.036

Fassò, A. (2013). Statistical assessment of air quality interventions. Stochastic Environ. Res. Risk Assess., 27(7), 1651-1660. https://doi. org/10.1007/s00477-013-0702-5

GBMW. (The Green-Breath Monitoring Website) (2016). Hourly AQI Monitoring Data. http://www.pm25.com/city/xiangtan.html/

Ge, B.Z., Cui, P., Lu, Q.N., and Wei, Y.L. (2008). Implement of phase unwrapping of digital holographic reconstruction image by discrete cosine transform least square method. J. China Univ. Pet., 32(6), 169-173.

Grell, G. and Baklanov, A. (2011). Integrated modeling for forecasting weather and air quality: A call for fully coupled approaches. Atmos. Environ., 45(38), 6845-6851. https://doi.org/10.1016/j.atmo senv.2011.01.017

He, G. and Deng, Q. (2012). A hybrid ARIMA and neural network model to forecast particulate matter concentration in Changsha, China, Proc. of the 10th International Conference on Healthy Buildings, Brisbane, QLD, Australia, 1, 242-247.

Heaton, J. (2005). Chapter 5: Understanding Back Propagation in Heaton, J. (Ed.): Introduction to Neural Networks with Java. Heaton Research, Inc., St. Louis, MO, ISBN 097732060X, $978097-$ 7320608, pp.125-154.

Kolehmainen, M., Martikainen, H., and Ruuskanen, J. (2001). Neural networks and periodic components used in air quality forecasting. Atmo. Environ., 35(5), 815-825. https://doi.org/10.1016/S1352-23 10(00)00385-X

Konovalov, I.B., Beekmann, M., Meleux, F., Dutot, A., and Foret, G. (2009). Combining deterministic and statistical approaches for PM10 forecasting in Europe. Atmos. Environ., 43(40), 6425-6434. https://doi.org/10.1016/j.atmosenv.2009.06.039

Kukkonen, J., Partanen, L., Karppinen, A., Ruuskanen, J.,Junninen, H., Kolehmainen M., Niska, H., Dorling, S., Chattertonc, T., Foxalld, R., and Cawley, G. (2003). Extensive evaluation of neural network models for the prediction of $\mathrm{NO}_{2}$ and PM10 concentrations, compared with a deterministic modelling system and measurements in central Helsinki. Atmos. Environ., 37(32)45394550. https://doi.org/10.1016/S1352-2310(03)00583-1

Kumar, A. and Goyal, P. (2011).Forecasting of daily air quality index in Delhi. Sci. Total Environ., 409(24), 5517-5523. https://doi.org/ 10.1016/j.scitotenv.2011.08.069

Liu, F., Wen, Z., and Zheng, L.J. (2015). Mathematical modeling for air quality management under stochastic and interval uncertainties. Stochastic Environ. Res. Risk Assess., 29(5), 1485-1498. https:// doi.org/10.1007/s00477-014-0934-z

Niska, H., Karppinen, A., Ruuskanen, J., and Kolehmainen, M. (2004). Evolving the neural network model for forecasting air pollution time series. Eng. Appl. Art. Intell., 17(2), 159-167. https:// doi.org/10.1016/j.engappai.2004.02.002

Plaia, A., Di Salvo, F., Ruggieri, M., and Agró, G. (2013). A Multisite-Multipollutant Air Quality Index. Atmos. Environ., 70: 387-391. https://doi.org/10.1016/j.atmosenv.2013.01.028

Rahman, N.H.A., Lee, M.H., Latif, M.T., and Suhartono, S. (2013). 
Forecasting of air pollution index with artificial neural network. $J$. Teknol., 63(2), 59-64. https://doi.org/10.11113/jt.v63.1913

Sayood, K. and Borkenhagen, J. (1991). Use of residual redundancy in the design of joint source/channel coders. IEEE Trans. Commun., 39(6), 838-846. https://doi.org/10.1109/26.87173

Sowlat, M.H., Gharibi, H., Yunesian, M., Mahmoudi, M.T., and Lotfi, S. (2011). A novel fuzzy-based air quality index (FAQI) for air quality assessment. Atmos. Environ., 45(12), 2050-2059. https:// doi.org/10.1016/j.atmosenv.2011.01.060

StatSoft, Inc. (2013). Electronic Statistics Textbook. Tulsa, OK: StatSoft. WEB: http://www.statsoft.com/textbook/ (accessed on April 1, 2014).

Sun, W., Zhang, H., Palazoglu, A., Singh, A., Zhang, W.D., Liu, S.W. (2013). Prediction of 24-hour-average PM2.5 concentrations using a hidden Markov model with different emission distributions in Northern California. Sci. Total Environ., 443, 93-103. https://doi. org/10.1016/j.scitotenv.2012.10.070

WHO. (2014). "7 million premature deaths annually linked to air pollution".

Wong, T.W., Tam, W.W.S.,Yu, I.T.S., Lau, A.K.H., Pang, S.W., and Wong, A.H.S. (2013). Developing a risk-based air quality health index. Atmos. Environ., 76, 52-58. https://doi.org/10.1016/j.atmosenv. 2012.06.071

Yang, Z.C. (2013). Fourier analysis Based Air Temperature Movement Analysis and Forecast. IET Signal Proces, 7(1), 14-24.
Yang, Z.C. (2014a). Modeling and forecasting daily movement of ambient air mean PM2.5 concentration based on the elliptic-orbit model with weekly quasi-periodic extension: A case study. Environ. Sci. Pollut. Res., 21(16), 9959-9972. https://doi.org/10.1007/s11356014-2899-3

Yang, Z.C. (2014b). Modeling and Forecasting Monthly Movement of Annual Average Solar Insolation Based on the Least-Squares Fourier-Model. Energy Convers. Manage., 81:201-210. https://doi.org/ 10.1016/j.enconman.2014.02.033

Yang, Z.C. (2016). DCT-based predictive model extended in the least-squares sense for hourly load forecasting. IET Generation Trans. Distrib., 10(15), 3930-3939. https://doi.org/10.1049/iet-gtd. 2016.0689

Yang, Z.C. (2017). Predictive modeling of hourly water-level fluctuations based on the DCT least-squares extended mode. Water Resour. Manage., 2017. https://doi.org/10.1007/s11269-017-1858-4

Yu, L., Zhao, Y. and Tang, L. (2014). A compressed sensing based AI learning paradigm for crude oil price forecasting. Energy Econ., 46, 236-245. https://doi.org/10.1016/j.eneco.20 14.09.019

Zhang, Y., Bocquet, M., Mallet, V., Seigneur, V., and Baklanov, A. (2012). Real-time air quality forecasting, part I: History, techniques, and current status. Atmos. Environ., 60:632-655. https://doi. org/10.1016/j.atmosenv.2012.06.031

Zheng, J.L., Ying, Q.H., and Yang, W.L. (2011). Signals \& Systems (3rd Edition). China Higher Education Press, Beijing 